\title{
CONFERENCES AS VIRTUAL ENTERPRISES
}

\author{
Jürgen Dorn, Peter Hrastnik, and Albert Rainer \\ Electronic Commerce Competence Center (EC3), \\ Donau-City-Straße 1, A-1220 Wien, AUSTRIA, \\ Email: \{juergen.dornlpeter.hrastniklalbert.rainer\}@ec3.at
}

\begin{abstract}
During the organization of a conference and moreover during the conference a cooperation between different organizations is necessary to deliver optimal services for the conference participants. In the $m>$ Forvm project we apply techniques developed for virtual enterprises in the MOVE project. $m>$ Forvm addresses optimized communication to participants as well as to supporting organizations. The integration between organizations is built on web services whereas the communication to conference participants is multi-modal. The business model "scientific conference" and its business processes are modeled with ProSpec, a graphical modeling tool. Communication processes and other workflows modeled are translated into XPDL (a workflow language) and deployed as web services. These XPDL processes are then executed and controlled by a web based process management system (WPMS). The framework and some described communication processes are evaluated at a large congress of radiologist in Vienna this year.
\end{abstract}

\section{INTRODUCTION}

ec3 (www.ec3.at) is a research center funded by different industrial, governmental and academic organizations. The research was organized until recently very traditional. Different projects were defined with certain research challenges in which typically two partners (one industrial and one research group) worked together.

With $\mathrm{m}>$ Forum a project was started that brings together competence from different industrial partners (a telecom partner, an ERP partner, as well as partners from conference organisation and tourism) and different basic research projects (projects on user interface design, data and web mining, cluster analysis, business modelling and evaluation, mobile services and virtual enterprises) of ec3.

The organization of the project can be seen as a virtual organization that produces a system by collaborating in different areas. Due to the different topics and partners the project coordination is more difficult than other projects. Special methods and tools are used such as tools for collaboration. Moreover, the output addresses a virtual organization or enterprise itself. 
The formal objective was to found a project/product that has a high innovative character and can be realized with the given competencies. The result was the definition of $m>$ Forvm. The project shall support conferences with innovative solutions in the area of multi-modal communication (i.e. mobile and Internet communication as well as local communication techniques such as Bluetooth or radio frequency technology). The system shall support the organization and performance of conferences. The main service such an organization offers to its customers (conference participants and the companies/organizations for which the participants work) is education. Thus a conference organizer must evaluate requirements of its clients (what knowledge need organization and participant) and must search for partners that collaborate in the production of the required knowledge and other services. Two functions of a virtual enterprise achieve special attention in the project: the communication and cooperation and the personalization of services.

In the next section we analyze conferences process-oriented without going into much detail. In the third section we sketch the framework for virtual enterprises used to deploy the first prototypes. In the fourth section we describe examples for the improved communication and in the fifth section the personalization issue is addressed.

\section{ROLE AND PROCESS ANALYSIS FOR A CONFERENCE}

We have analyzed the different roles participating in a conference and which objectives these roles have. Based on this organizational model, we conduct a rigorous process-oriented analysis of such an organization consisting of

- an identification of roles and required services,

- the required processes and subfunctions to obtain the services,

- the information flow and required data, and

- other required resources.

One important role can be classified as conference organization where we can identify subroles of:

- a program chair (PC) who is responsible for the paper selection subprocess,

- a local organizer (LO)who is responsible for organizing the local resources,

- a professional conference organisation (PCO) which is responsible for typical arrangements for conference participants and

- one or more scientific organisations (SO) such as IFIP that issue certain quality standards and policies for conferences.

The most important role is the participant which is the main target for finding innovative services and improvements. Additionally, we regard the relationship between conference organization and the originating organization for which the participant works. This organization typically pays for the participant and an improved billing process is an achievement that can be realized without great effort.

An objective more ambitious is the support of the human resource management of originating organizations. An organization's objective may be to plan more detailed the required knowledge for the organization and its staff. On one side, better 
information which knowledge assets can be learned at a conference is to be delivered to an organization and on the other side, we could supply the organization with information which knowledge assets the participant has achieved during a conference.

Another role is the exhibitor that presents products and services related to the scientific program of the conference. An exhibitor is interested to get contact data from participants. Here we must regard privacy aspects. However, there could be benefits achieved with electronic communication. A similar role is the sponsor who has usually the interest to make publicity and wants to have the sponsor's brand related to a prestigious conference. The role destination stands for local authorities that see a large conference as a possibility to attract more tourists to a region. Supporting organisations are those organisations that deliver resources for a conference such as rooms, transportation, catering and cultural events.

The following figure gives an abstract view on roles delivered by the business process "performingConference".

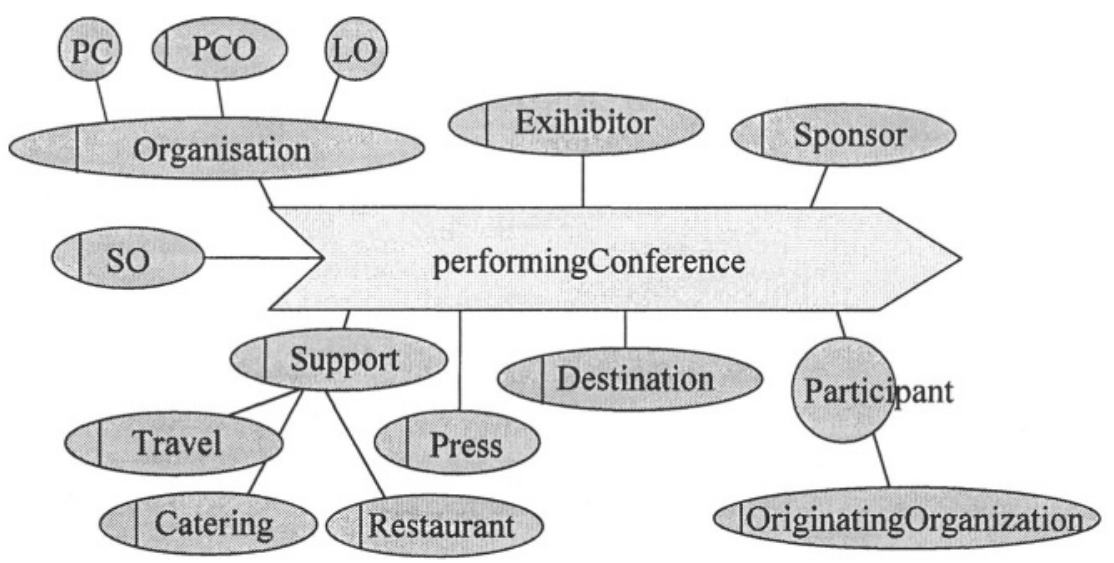

Figure 1: Participating roles and services expected

Derived from the described roles we can identify expected services and from that objectives for process improvements for each role. There are many services produced by a conference but the most important should be knowledge or education of participants. We must address our innovative developments especially to the improvement of the education aspect. We identify three sub points:

- A participant learns by attending a talk, reading a paper, and maybe by discussing with the speaker. The objective for the conference organization is to get the best speakers able to present required knowledge and indicating to participants which presentation fit their interests best.

- A participant may improve his capabilities by giving a talk. The objective here is to help in the preparation and evaluate the talk to enable continuous improvement of the speakers capabilities.

- An important service is to make contacts with either VIPs in an area or with colleagues working in a similar environment. The objective is to 
match interests and enable easy communication and establishment of contacts.

Further services produced by a conference are publicity and profit. Supporting organization and PCOs are interested in profit and other roles are interested in publicity which will also improve the value of the conference.

The next step is to make the process analysis to detect potential opportunities for improvements. Special attention was paid into improvements by mobile communication. The following figure shows the main business processes.

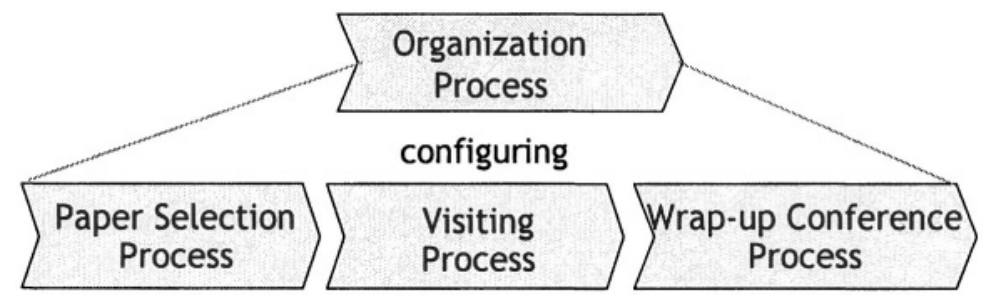

Figure 2: Main Processes of Conference

The organizing process has the following typical activities:

- financial planning,

- planning resources such as conference rooms, hotels, ...,

- communication with exhibitors, sponsors and press,

- planning the paper selection process and selection of reviewers,

- planning quality management.

These activities show that this process determines features of other processes. The potential for mobile communication is low here. However, an improvement could be an electronic search for resources such as rooms and invited speakers.

The first operative business process is the paper selection process containing the following activities:

- submission of abstracts, papers or posters,

- assignment of reviewers to submissions,

- reviewing (several variants are possible here),

- $\quad$ selection of papers or posters,

- $\quad$ assigning talks into sessions and scheduling sessions.

The potential of mobile communication is also in this process low. Moreover, there already exist some good software packages that support this process. The process could be extended by an SMS-notification service, but it is anticipated that such a service will seldom be used. However, the process may give us a lot of inputs required later for services delivered with mobile communication. For example, from a classification of papers we derive partial interests of participants that may be used to notify the participant about similar talks.

The greatest potential for mobile communication exists in the visiting process. Since the participant has left his/her usual working environment s/he is more 
dependent on mobile resources. We must distinguish different mobile devices the participant may use. This may be a laptop, a PDA or a simple mobile phone. Some of the activities that may be supported by mobile services are:

- registration,

- arriving at airport and transfer to hotel or conference venue,

- check-in at conference desk and information delivering,

- finding of interesting talks and session (push or pull-service)

- making contacts with other participants,

- searching other participants and notification of changes,

- evaluation of talks, sessions, posters, exhibitions and the whole conference,

- information on local events, transportation and similar,

- reservation of restaurants and cultural events,

- organizing social events ad-hoc.

This shows where the greatest benefit of mobile communication may exist. The last process is the "Wrap-up Conference Process". The following activities must be performed here:

- financial clearing,

- conference evaluation and detection of possible improvements,

- after conference contacts,

- production of proceedings (may also be performed before the conference takes place) and other media from the conference content

- planning of next conference.

\section{BUSINESS PROCESS MODELING AND EXECUTION}

The MOVE project, one of ec3's basic research projects, aims at an integrated development environment for virtual enterprises. This development environment is applied in $\mathrm{m}>$ Forvm. Three subareas are investigated:

- modelling of organization and processes in virtual enterprises,

- mechanisms for optimized workflows and communication in virtual enterprises and

- optimization of resources in virtual enterprises.

One of the most important functions in a virtual enterprise is the communication between partners and the customers. First step in the development process is the definition of the desired business model. The second step is the definition of business processes such as for example "performingConference". Business processes may be defined hierarchically and one of the subprocesses will be "registratingParticipant". On the lowest process level, we have technology driven processes such as "exchangingDataWithMobilePhone" or "queringDatabaseForMembership". Whereas the high level processes are very specific for a new business model, the low level processes can be reused easily by parametrizing. For our example, we must use specific data that is exchanged between process and phone or which organization we ask for membership.

A process model may contain several activities to be performed and a control flow describing the choreography of the process. An activity may be a sub process, a 
web service call, or some other action performed either by another program or by a human. Our main focus in the project, however, is on web services based architecture (W3C, 2003). Besides the control flow, the information flow is specified by defining data records and flow between activities. Here, we specify which data is to be queried from the customer and which data is to be read from a database. The business model as well as the hierarchically defined processes are modelled with ProSpec a Java-based graphic process modelling tool (Rainer, 2004).

The modelled process can be stored as an XPDL-process. XPDL (WFMC, 2002) is a XML-based language proposed by the Workflow Management Coalition. XPDL enables to describe the control flow in a process and can be easily extended to integrate also web services. Processes can be stored in a ebXML compliant repository or deployed itself as a web service.

OBE (Open Business Engine) (Eden, 2003) is a workflow management system that executes and controls processes specified in XPDL. We have extended OBE by web service functionality enabling us to execute processes as described. The new Web-based Process Management System (WPMS) supports also transactions which is especially important in open communication systems where no guarantee for answers can be given.

The WPMS is used for example to execute and control the registration process for a conference participant. The registration process is deployed as web service. This web service calls another web service that is responsible for checking whether the participant is member of supporting scientific organization. The registration service can be called by a simple http request in any Internet browser. Moreover, with the software described in (Dorn and Sillhengst, 2003) this service can also be called by a Java-enabled mobile phone.

For typical functions occurring in a virtual enterprise we have defined so called infrastructure web services, An example is the querying of a database.

\section{COMMUNICATION}

As already mentioned, the communication is one of the most important technical aspects that must be addressed in a virtual enterprise. Our approach is on developing basic building blocks (processes and web services mainly) for a virtual enterprise that are stored in a repository and can be reused easily. The ProSpec tool gives us the possibility to control the reuse process and to validate new configurations of services. In the following we describe briefly some communication processes already deployed or planned for the $m>$ Forvm project.

\subsection{Querying Membership in scientific organization}

Usually members of scientific organizations get a reduction on the conference fee. To compute the fee applicable for a new participant, the membership database of the scientific organization may be queried. We have implemented an own database for membership in ec3 with a web service interface, and web service interfaces to the membership databases of the Austrian computer society and the European radiologist society. 
If a participant has filled out the registration form and claims a membership, it is evaluated whether an access via web service can be made to the database. If this check is positive, we call the web service with the participants name and obtain an answer whether membership is still active.

We could imagine that in future each organization supports such a web service interface which would make our registration process applicable for any conference.

\subsection{Reservation of restaurant}

If participants from foreign countries attend a conference it is often not so easy to use local services as for example reservation of tables in a restaurant. Typically local organizations support participants at the conference desk with such services. We collaborate with lokaltipp.at a company organizing restaurants for international guests at Austrian conferences. We improve the reservation process with electronic communication.

The participant may now specify his reservation inquiry in a web form, as an SMS-message, or at the conference desk. Since this inquiry cannot be handled immediately we offer the possibility that the participants receives the notification of a reservation by email or SMS. Thus the participant must not return to the conference desk or to the Internet site. The implementation of the service is as follows: The inquiry is sent by a web service call to an applet on the server of lokaltipp.at. This applet enables to manage different inquires in parallel. If a restaurant was found (by human phone call at lokaltipp.at side), the information is entered into the applet interface and the web service call returns the entered data to the reservation process at the conference side. Dependent on the participants preferences either an SMS or an email is sent to him. This reservation process can easily be extended to other resources which must be reserved. It is anticipated that eventually restaurants offer own web services for reservation that can be called by our process so that we can avoid the manual work of lokaltipp.at in future.

\subsection{Communication with participants company}

Another communication process which promises considerable improvements is the payment of conference fees and the transfer of the accounting information. We plan to integrate the conference processes with standard enterprise resource planning (ERP) software to facilitate the process of submitting data to the company of the participant. This communication will be realized again by web services. For participants working for organizations that are not interested in this direct communication, a service to produce easily recipes shall be incorporated in this process, too.

\subsection{Communication with exhibitors}

Exhibitors usually have stands at a conference where they present products that can be somehow associated with the scientific theme of the conference. For example, on a medical conference, pharmaceutical industry will present new products. However, typically not all products are important for every participants. Thus the exhibitor is interested to obtain contact data of those participants that have certain interests. Whether such contact data may be supplied depends of course on the permission of the participant. 


\section{PERSONALIZATION}

Better services to customers of virtual enterprise and also for participants of a conference can be achieved if every customer is offered individualized services. To achieve this we need to know interests and preferences of each customer. A virtual enterprise concept improves the capabilities of personalization, because all participating members may collect data of customer's behaviour in a similar manner. The whole enterprise obtains therefore more data which can be analyzed.

We use the following methods to improve our knowledge about each participant:

- participants may fill out forms in the registration process to express explicitly certain preferences

- $\quad$ keywords are extracted automatically from papers submitted and papers and with that their authors are classified

- participants are tracked during the conference with radio frequency technology to derive from their behaviour certain interests, and

- $\quad$ extracting knowledge from web sites through LiXto-technology.

\section{CONCLUSION}

The $m>$ Forvm project addresses fundamental improvements for the organization of conferences by new communication processes. Especially the better communication and cooperation between different organizations participating in the service production process will enable a personalized service for participant. Different preferences of participants will lead to different services delivered.

The main service is and will be new knowledge in a certain scientific domain. By better classifying papers and talks and by collecting interests and preferences of participants we will improve the efficiency of human learning conferences.

The project is not yet finished. In march a first large conference is used to evaluate how far offered services are accepted by participants. This will give us also new insights of future extensions.

\section{REFERENCES}

1. Dorn, J. m>Forum - Supporting conference organisations and participants with multi-modal communication, Technical Report TR-EC3-mForum-1, 2004.

2. Dorn, J. and Sillhengst, Th., 5th Annual Global Information Technology Management (GITM) World Conference, San Diego, 2004.

3. Eden, A, http://www.openbusinessengine.org/ (last visited March 4,2003)

4. Hrastnik, P. (2004) Business Processes Based on Web Services, Int. J. Electronic Business, Vol. 2.

5. Rainer, A. (2004) Web-Centric Business Process Modelling, Int. J. Electronic Business, Vol. 2.

6. W3C. Web services, www.w3.org/2002/ws/ (last visited November 15,2003)

7. WFMC. Workflow Management Coalition (2002), http://www.wfmc.org/standards/docs/TC1025_10_xpdl_102502.pdf (last visited March 4,2003) 